\title{
Effect of Selenium nanoparticles in wound healing
}

Keshta A.T..$^{(1)}$, Emam M..(2) Attia Y.A ${ }^{(3)}$

(1) Assistant professor of Biochemistry, Chemistry Department, Faculty of Science, Zagazig university

(2) Biochemistry division, Chemistry Department, faculty of Science, Zagazig Univeristy.

(3) Assistant professor of Physical chemistry, National Institute of Laser Enhanced Sciences, Cairo University

\begin{tabular}{ll}
\hline A R T I L E IN F & A B T R A C \\
\hline Keywords: & Background: Wound healing is a complex process \\
Wound healing & necessary to repairing damaged tissues and preventing \\
Antioxidant & infection. Selenium nanoparticles were known by their \\
Selenium nanoparticles & antioxidant and antimicrobial effect that is important in \\
& wound healing. Aim: the aim of this study was to \\
& investigate the effect of selenium nanoparticles in \\
& reducing and accelerating the wound healing time in \\
& mice. Methods: Se NPs were synthesized by a simple wet \\
& chemical method. Then the experimental animals were \\
& divided into the following groups: Negative control \\
& group, Positive control group, selenium nanoparticles \\
& control group, wounded mice treated with selenium \\
& nanoparticles group. At the end of experiment, mice were \\
scarified \& skin tissue samples were collected for \\
biological analysis [Vascular Endothelial cell Growth \\
Factor, Collagenase I and Nitric oxide] and \\
histopathological study. Results: The results showed that \\
the percentage of wound area has been significantly \\
reduced in Se NPs treated group compared to the positive \\
control. The level of Vascular Endothelial Growth Factor \\
and Collagenase I in Se NPs groups were significantly \\
increased compared to positive control while nitric oxide \\
was significantly decreased in Se NPs treated group. Skin \\
tissue showed effect of selenium nanoparticles by \\
regenerating and recavering skin layer. Conclusion: \\
Selenium nanoparticles have an important role in \\
accelerating and reducing wound healing time. \\
\hline
\end{tabular}

\section{Introduction:}

The skin forms an effective barrier between the body and external environment. If the protective skin barrier is disrupted, wound healing process is immediately begun by activating an orchestrated cascade of events ${ }^{(1)}$. Wound healing process can be divided into three or four overlapping phases: (1) hemostasis phase (some authors not considered this a phase) (2) inflammatory phase, (3) proliferative phase or new tissue formation (neoangiogenesis, proliferation, re-epithelialization); and (4) tissue 
remodeling (remodelling of extracellular matrix, ECM) ${ }^{(2)}$. Depending on growth factors, cytokines, and chemokines involved in a complex integration of signals that coordinate cellular processes lead to the success of the wound healing process ${ }^{(3)}$. Nanoparticles can be combined into biomaterials and scaffolds to form nanocomposite smart materials, which can help wound healing by their antimicrobial, selective anti- and pro-inflammatory, and pro-angiogenic properties. In addition, they can affect the healing process by influencing collagen deposition and realignment (4). For example Selenium (Se) metal that is one of the important trace mineral that plays an essential role for human health. Due to Selenium nanoparticles biocompatibility and low toxic nature as a result to its high specific surface area, high surface activity, abundant surface active centers, high catalytic efficiency and adsorbing ability, Se-NPs receiving a great attention ${ }^{(5)}$. This claimed support the aim of this study to evaluate the role of selenium nanoparticles in accelerating and reducing the wound healing time.

\section{Material and Methods: \\ Chemicals:}

VEGF and Collagenase 1 kit were obtained from New Test Co.Egypt. Nitric oxide kit was obtained from Biodiagnostic Co. Egypt. All chemicals used in preparation of Se-NPs were obtained from Sigma-Aldrich and used without further purification.

\section{Preparation of Se Nanoparticles}

$0.1 \mathrm{~g}$ of Polyvinylpyrrolidone (PVP) was dissolved in $40 \mathrm{~mL}$ of distilled water under stirring and $0.6 \mathrm{~g}$ of ascorbic acid was added to that solution and continuous stirring for $5 \mathrm{~min}$. $1 \mathrm{~mL}$ of $0.96 \mathrm{~g} / \mathrm{mL}$ of cetyltrimethylammonium chloride (CTAC) solution was added. After $5 \mathrm{~min}$ of stirring $1 \mathrm{~mL}$ of $0.25 \mathrm{M}$ of Sodium selenite was added drop-wise till the color of the solution changed from colorless into orange color (6). Transmission electron microscopy
(TEM) and UV-Vis absorption spectroscopy techniques have been employed to characterize the synthesized Se NPs.

\section{Characterization of Se NPs:}

Transmission electron microscopy (TEM) images were obtained with a Joel JEM-1230 electron microscope operated at $120 \mathrm{kV}$ equipped with Gatan Ultra Scan 4000SP 4K 9 4KCCD camera. A drop from diluted sample dispersion was deposited onto anamorphous carbon film on 400 mesh copper grids and left to evaporate at room temperature. UV-Vis spectra were measured with a Perkin Elmer Lambda 40 UV-visible spectrophotometer using 1-cm path length Hellma quartz cuvettes.

\section{Animals:}

Forty female albino mice, each weighed $25-30 \mathrm{~g}$ with age (2 month \pm week) were, were housed in special cages (10 - mice/cage) and maintained under controlled environmental conditions (12-hours light/dark cycle, temperature $27^{\circ} \mathrm{C}$ ) in animal house of Faculty of Science - Zagazig University, and provided with standard laboratory food and water ad libitum. In accordance with the guide for the care and use of laboratory animals all studies were performed, as adopted and promulgated by the Institutional Animal Care Committee [ZU-IACUC/1/F/117/2018].

\section{Wound model}

Mice were anesthetized by light dosage of diethyl ether and then 250 $\mathrm{mm}^{2}$ full thickness open excision wound was opened at the lower right part of the dorsum ${ }^{(7)}$.

\section{Experimental design:}

The animals were divided into four groups each one contains 10 mice as following:

Group I: Negative control group, injected with saline along experiment.

Group II: Positive control group, wounded mice $\left(250 \mathrm{~mm}^{2}\right.$ full thickness open excision wound). 
Group III [Se-NP control group]: healthy mice were injected intraperitoneal (IP) with Se-NP (0.5 $\mathrm{mg} / \mathrm{kg}$ ) daily for 30 day ${ }^{(8)}$.

Group IV [Se-NP treated group]: wounded mice were injected IP with Se$\mathrm{NP}(0.5 \mathrm{mg} / \mathrm{kg})$ daily for 30 day.

2.6. Wound contraction

Digital photographs of wounds were taken at different times $(0,7,15,23,30$ days) for following the difference and speed of wound healing process in treated group compared with positive group.

\section{Sample collection}

\section{-Tissues homogenate:}

At the end of experiment, skin tissues of wound area were obtained to form homogenate. Samples were homogenized in $4 \mathrm{ml}(0.1 \mathrm{~g}$ tissue) of cold buffer (50mM phosphate buffer, $\mathrm{pH} 7.0$, containing $5 \mathrm{mM}$ EDTA, and $1 \mathrm{mM} 2-$ mercaptoethanol) and then separated in centrifuge at $3000 \mathrm{rpm}$ for $10 \mathrm{~min}$ at $20 \mathrm{c}$ (9).

\section{- Biochemical Analysis:}

Vascular endothelial growth factor (VEGF), Collagenase I and Nitric oxide were determined according to method described by ${ }^{(10-12)}$ respectively.

\section{-Histopathological study}

Skin tissues were removed and preserved in $10 \%$ formalin for fixation, then embedded in paraffin cubes. Thin sections (4-5 m) were stained with hematoxylin and eosin ( $\mathrm{H} \& \mathrm{E})$ solution and examined under light microscope power of (X 100 -400) for histopathological examination ${ }^{(13)}$.

\section{Statistical analysis:}

Data were statistically analyzed for descriptive measurements including arithmetic mean and standard error using SPSS program version 23. The one-way ANOVA test was performed for comparison between the different groups [i.e. Negative, Positive, Se (C), Se (W) ] and followed by post hoc test using Duncan multiple rang (DMR) test for comparisons between means of groups.
The means followed by the same letter in each column are not significantly different from each other at the 5-percent probability level $\mathrm{P}^{(14)}$.

\section{Results}

\section{Characterization of Selenium nanoparticles}

Ascorbic acid was used as a reducing agent because it is biocompatible and has good reducing properties forming a spherical nanoparticle having average size $30.22 \pm 1.21 \mathrm{~nm}$ as measured using TEM in Fig. 1. Nanoparticles were stabilized by the presence of PVP and CTAC. When sodium selenite was allowed to react with ascorbic acid, the selenium was reduced to elemental selenium $\left(\mathrm{Se}^{0}\right)$. The color change from colorless to orange indicated the occurrence of the reduction reaction to form selenium nanoparticles. Such a color change of $\mathrm{Se}$ nanoparticles dispersion could be more clearly manifested by the UV-Visible absorption spectra in Fig. 2. Se NPs shows maximum absorbance at $259 \mathrm{~nm}$.

\section{Effect of Se-NP on wound area}

Photographing of wound healing process in mice at different time points (Figure 3 ), showed that the wound area was highly reduced in Se-NP treated group compared to positive control group.

Effect of Se-NP on vascular endothelial growth factor in the skin tissues of all studied groups

Table (1) and figure (4) demonstrated the effect of Se-NP on VEGF level in the skin tissues of all studied groups.

The mean values of VEGF (pg/g tissue) were increased from $12.16 \pm 0.24$ in negative control group (G I) to $24.61 \pm$ 0.28 in group (G II) by $102.47 \%$. There was significant decrease in (G IV) treated group and (G III) control group by $-16.03 \%$ and $-88.12 \%$ respectively compared to (G II) and (G I).

Effect of Se-NP on collagenase 1 activity in the skin tissues of all studied groups 
Table (1) and figure (4) demonstrated the effect of Se-NP on collagenase 1 activity in the skin of all studied groups.

The mean values of collagenase 1 (ng/g tissue) were increased from $1.22 \pm 0.08$ in negative control group (G I) to $3.86 \pm$ 0.1 in group (G II) by $217.84 \%$. There was significant decrease in (G IV) treated group by $-84.06 \%$ in comparison with (G II). While there was nonsignificant decrease in (G III) control group by $-9.09 \%$ in comparison with $(\mathrm{G}$ I).

\section{Effect of Se-NP on nitric oxide (NO) concentration in the skin tissues of all studied groups.}

In skin tissues as showed in Table (1) and figure (4) the mean values of NO ( $\mu \mathrm{mol} / \mathrm{g}$ tissue) were increased from 4.12 \pm 0.30 in negative control group (G I) to $16.07 \pm 0.17$ in group (G II) by $290.38 \%$ . There was significant decrease in $(\mathrm{G}$ IV) treated group and (G III) control group by $-44.9 \%$ and $-25.20 \%$ respectively in comparison with (G II) and (G I).

\section{Histological changes}

Histological study was done for the skin tissues of scarified female albino mice from all groups after healing to evaluate the impact of Se-NP on skin during healing process.

As shown in figure (5): Skin tissue from positive control group (G II) (A) was uncompleted wound healing. There were observed changes in (G IV) (B) treated group, showed that there was regeneration effect on wounded skin by forming skin layers. Skin tissue from negative control group (G I) (A") showed normal skin layers. But Skin tissue from (G III) (B") control group showed few changes compared to (G I) like edema in the dermis layer.

\section{Discussion}

Wound has been defined as a disruption of normal anatomical structure and function. Therefore, healing is the complex and dynamic process that lead to the restoration of anatomical continuity and function ${ }^{(15)}$. Although the wound healing process take place spontaneously and does not need much help, there are several risk factors such as infection, supply of blood, nutritional status and other factors that impact the resolution of this process ${ }^{(16)}$. It is well known that the natural wound healing process was delayed by microbes attacking which invade the skin barrier (17). The vascular endothelial growth factor (VEGF) is predominantly secreted by keratinocytes located at the wound site (18). VEGF is a regulator of angiogenesis and stimulates endothelial cell functions needed for new blood vessel formation, such as proliferation, migration, differentiation, and survival (19). Also Matrix Metalloproteinase like collagenase 1 (MMP-1), is the major human interstitial collagenase, might play a significant role in fibrous plaque disruption by contributing to the degradation of interstitial collagens and thinning of the fibrous cap ${ }^{(20)}$. NO play an important role in all phases of wound healing but it might increase the nitroxidative stress ${ }^{(21,22)}$. In this study, the level of NO in skin tissues was increased in Positive control group in response to injury of the skin. While their level were decreased in Se-NP treated group, this may be due to the antioxidant activity of Se-NP where SeNP with their small size and large surface area had more atoms exposed to free radicals for the electron exchanger, with a high potential for scavenging multiple free radicals (23). This result were in agreement with Dkhil et al. ${ }^{(24)}$, who indicated that SeNPs able to decrease the levels of nitric oxide and lipid peroxidation which lead to reducing oxidative stress and apoptosis. Also the data showed that the level of VEGF and collagenase 1 in Se-NP treated group of skin tissues were lower than that of Positive control group, the lower level showing the ending of wound healing process as at the terminal stages of 
healing angiogenesis is suppressed, growth factor levels decline as tissue normoxia is restored and inflammation subsides ${ }^{(25)}$. The results of histological examination of the skin were confirmed the biochemical analysis. Se-NP treated group showed regeneration of skin layer in skin tissue compared to positive control group, this indicate proliferation of basal layer of epidermis to compensate skin loss in response to wound healing effect of Se-NP ${ }^{(26-29)}$.

\section{Conclusion:}

This study observed the remedial potential of Se-NP in wound healing and based on our results, we indicated that SeNP significantly stimulated wound contraction.

\section{References:}

1. Xue, M., \& Jackson, C. J. (2015). Extracellular Matrix Reorganization during Wound Healing and Its Impact on Abnormal Scarring. Advances in Wound Care, 4(3), 119-136.

2. Saghazadeh, S., Rinoldi, C., Schot, M., Kashaf, S. S., Sharifi, F., Jalilian, E., Khademhosseini, A. (2018). Drug delivery systems and materials for wound healing applications. Advanced Drug Delivery Reviews, 127, 138-166.

3. Barrientos, S., Stojadinovic, O., Golinko, M. S., Brem, H., \& TomicCanic,M.(2008). PERSPE CTIVE ARTICLE: Growth factors and cytokines in wound healing. Wound Repair and Regeneration, 16(5), 585-601.

4. Seifalian, A. M. (2018). Nanoparticles in wound healing from hope to promise from promise to routine. Frontiers in Bioscience, 23(3), 1038-1059.

5. Anu, K., Singaravelu, G., Murugan, K., \& Benelli, G. (2016). Green-Synthesis of Selenium Nanoparticles Using Garlic Cloves (Allium sativum): Biophysical Characterization and Cytotoxicity on Vero Cells. Journal of Cluster Science, 28(1), 551-563.
6. Vahdati, M., Moghadam, T.T., 2020. Synthesis and characterization of Selenium nanoparticles-Lysozyme nanohybrid System with Synergistic Antibacterial properties. Scientific Reports 10, 510.

7. Eldelghdye, S. M., AbdelKhaled,L. G., Taha, M. S., Allam, T. M., Hassanian, W. F., Keshta, A. T., Alfrarge, A. (2016). Role of Purslane (PORTULACA OLERACEA) in the wound healing and its impact on biochemical parameters in diabetic albino rats. International Journal of Biology, Pharmacy and Allied Sciences, 5(7), 1576-1590.

8. Hassanin KM, El-Kawi SHA, Hashem KS. (2013).The prospective protective effect of selenium nanoparticles against chromiuminduced oxidative and cellular damage in rat thyroid. Int J Nanomedicine, 8:1713-1720.

9. Noori S., Azmat M., Mahboob T., (2012): Study on antioxidant effects of cinnamon and garlic extract in liver, kidney and heart tissue of rat. Biosci. Res, 9, 17-22.

10. Darland, D. ., Massingham, L. ., Smith, S. ., Piek, E., SaintGeniez, M., \& D'Amore, P. (2003). Pericyte production of cell-associated VEGF is differentiation-dependent and is associated with endothelial survival. Developmental Biology, 264(1), 275-288.

11. Tokarzewicz, A., Romanowicz, L., Sveklo, I., \& Gorodkiewicz, E. (2016). The development of a matrix metalloproteinase-1 biosensor based on the surface plasmon resonance imaging technique. Analytical Methods, 8(34), 6428-6435.

12. Montgomery, H., A., $\mathrm{C}$ and Dymock, J., F. (1961). Analyst, 86, 414.

13. Slaoui, M., \& Fiette, L. (2010). Histopathology

Procedures: From Tissue 
Sampling to Histopathological Evaluation. Drug Safety Evaluation, 69-82.

14. IBM Corp. Released (2013). IBM SPSS Statistics for Windows, Version 22.0. Armonk, NY: IBM Corp.

15. Diegelmann, R. F., Evans, M. C. (2004). WOUND HEALING: AN OVERVIEW OF ACUTE, FIBROTIC AND DELAYED HEALING. Frontiers in Bioscience, 9, 283-289.

16. Peters EJ, Lipsky BA. (2013). Diagnosis and management of infection in the diabetic foot. Med Clin North Am; 97(5):911- 46.

17. Pattanayak SP, Sunita P. (2008). Wound healing, anti-microbial and antioxidant potential of Dendrophthoe falcata (L.f) Ettingsh. Ethnopharmacol;120(2):241-7.

18. Birkenhauer,E.,\&Neethirajan, S. (2015). A Double-Edged Sword: The Role of VEGF in Wound Repair and Chemoattraction of Opportunist Pathogens. International Journal of Molecular Sciences, 16(12), 7159-7172.

19. Johnson, K. E., \& Wilgus, T. A. (2014). Vascular Endothelial Growth Factor and Angiogenesis in the Regulation of Cutaneous Wound Repair. Advances in Wound Care, 3(10), 647-661.

20. Rohani, M. G., \& Parks, W. C. (2015). Matrix remodeling by MMPs during wound repair. Matrix Biology, 44-46, 113-121.

21. Blecher, K., Martinez, L. R., Tuckman-Vernon, C., Nacharaju, P., Schairer, D., Chouake, J., Friedman, A. J. (2012). Nitric oxide-releasing nanoparticles accelerate wound healing in NOD-SCID mice. Nanomedicine: Nanotechnology, Biology and Medicine, 8(8), 1364-1371.

22. Droge W. (2002). Free radicals in the physiological control ofcell function. Physiol Rev, 82, 47-95.
23. Wang, Yan, X., \& Fu, L. (2013). Effect of selenium nanoparticles with different sizes in primary cultured intestinal epithelial cells of crucian carp, Carassius auratus gibelio. International Journal of Nanomedicine, 8(1), 4007-4013.

24. Dkhil, M., Zrieq, R., AlQuraishy, S., \& Abdel Moneim, A. (2016). Selenium Nanoparticles Attenuate Oxidative Stress and Testicular Damage in Streptozotocin-Induced Diabetic Rats. Molecules, 21(11), 1517.

25. Tsala, D. E., Amadou, D., Habtemariam, S. (2013). Natural wound healing and bioactive natural products. Phytopharmacology, 4(3), 532560.

26. Ramya,S., Shanmugasundaram, T., \& Balagurunathan, $\mathbf{R}$. (2015). Biomedical potential of actinobacterially synthesized selenium nanoparticles with special reference to anti-biofilm, anti-oxidant, wound healing, cytotoxic and anti-viral activities. Journal of Trace Elements in Medicine and Biology, 32, 30-39.

27. Chung, S., Ercan, B., Roy, A. K., \& Webster, T. J. (2016). Addition of Selenium Nanoparticles to Electrospun Silk Scaffold Improves the Mammalian Cell Activity While Reducing Bacterial Growth. Frontiers in Physiology, 7.

28. Abbaszadeh,A., TehmasebiFoolad, A., Rajabzadeh, A., Beigi-Brojeni, N., Zarei, L. (2019). Effects of Chitosan/Nano Selenium Biofilm on Infected Wound Healing in Rats; an Experimental Study. Bull Emerg Trauma; 7(3):284-291.

29. Rostami, H., Mohammadi, R., Asri-Rezaei, S., Tehrani, A. (2018). Evaluation of Application of Chitosan/Nano Selenium Biodegradable Film on Full Thickness Excisional Wound 
Healing in Rats. Iranian Journal

of Veterinary Surgery; 13(1).
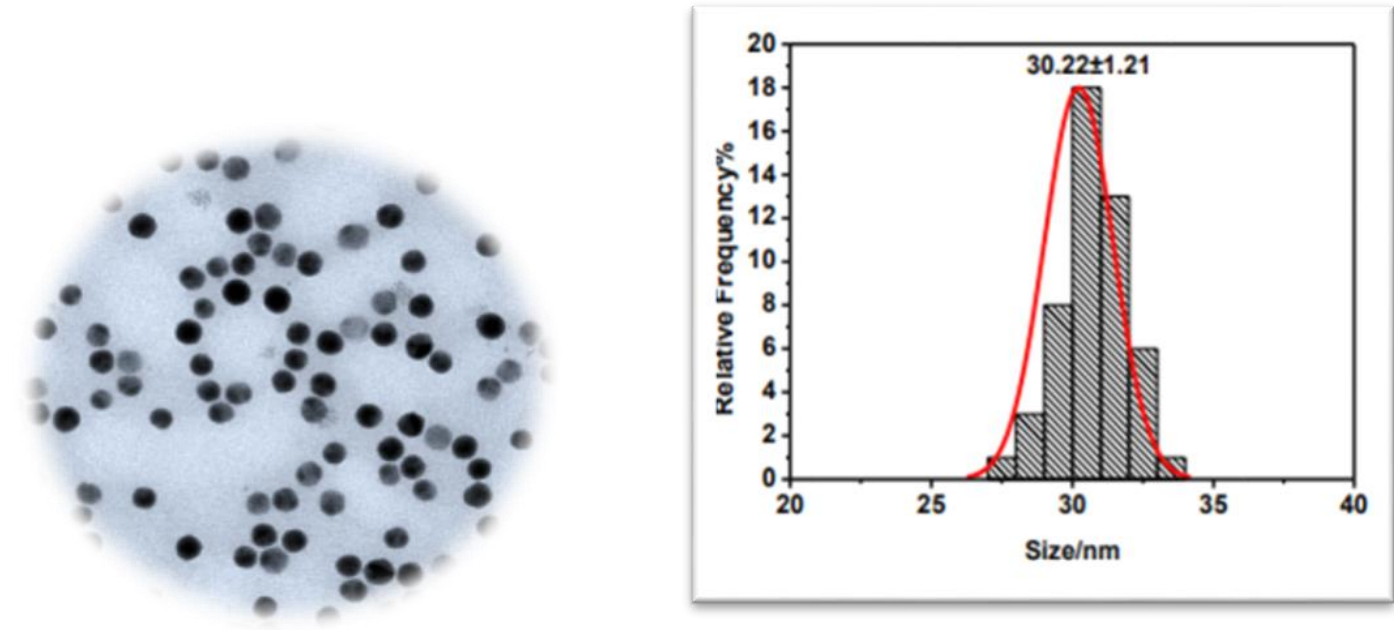

Figure (1): TEM and histogram images of water soluble Se nanoparticles being collected from the freshly synthesized Se NPs suspension.

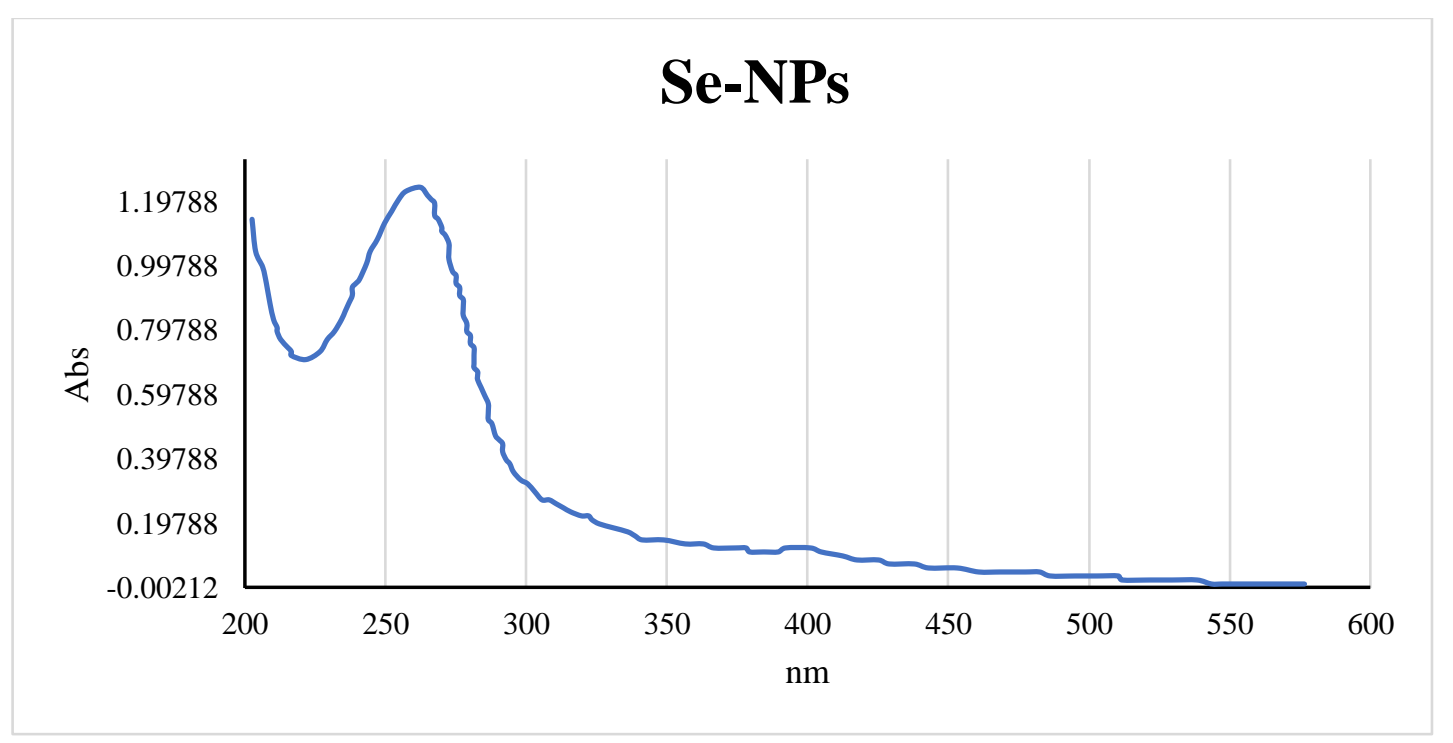

Figure (2): UV-visible optical spectra of Se NPs peak at $259 \mathrm{~nm}$. 


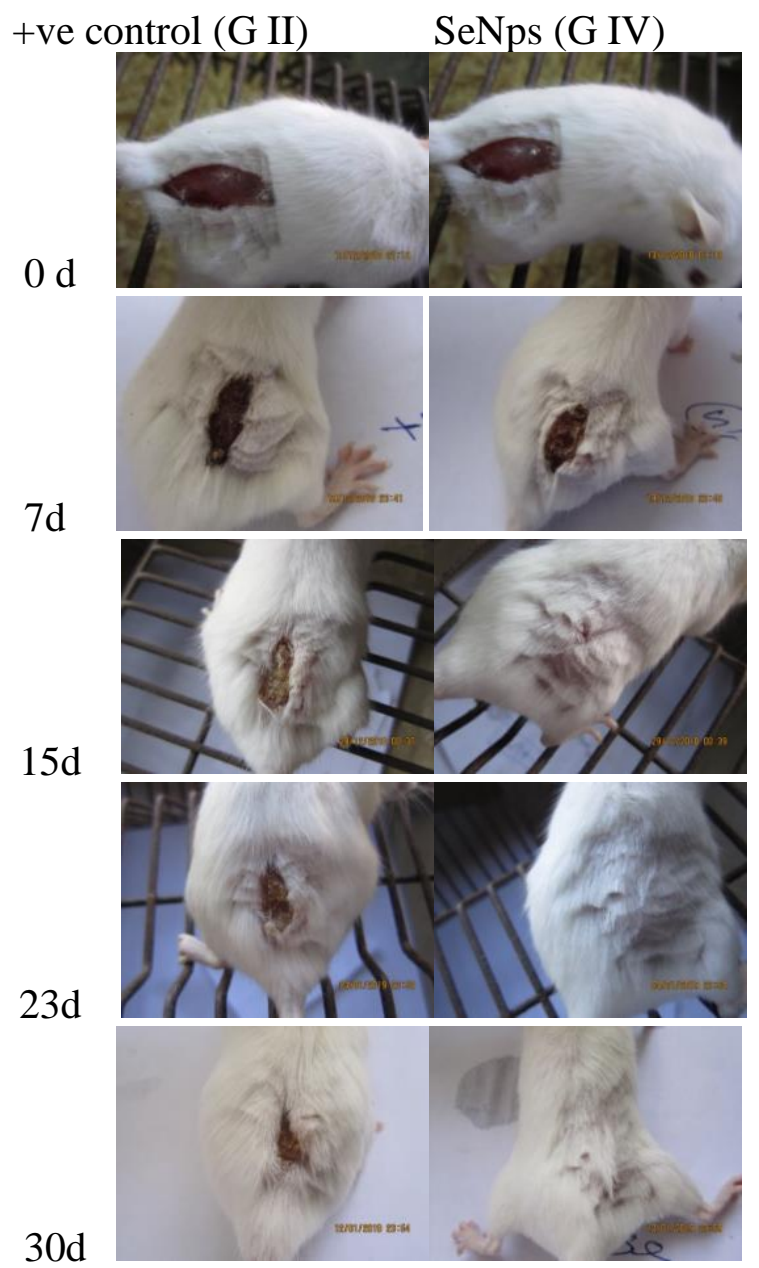

Figure (3): wound healing contraction at different times $(0,7,15,23,30$ days) in all studied groups in adult female albino mice.

Table 1. Vascular endothelial growth factor levels, Collagenase 1 activity and Nitric oxide (NO) concentrations in skin tissues of all studied groups.

\begin{tabular}{|c|c|c|c|c|}
\hline \multicolumn{2}{|c|}{ Groups } & VEGF & collagenase 1 & Nitric oxide \\
\hline \multirow{2}{*}{$\begin{array}{l}\text { Negative } \\
\text { Control } \\
\text { (G I) }\end{array}$} & Mean \pm SE & $12.16 \pm 0.24^{f}$ & $1.22 \pm 0.08^{\mathrm{d}}$ & $4.12 \pm 0.30^{\mathrm{e}}$ \\
\hline & \% Change & ---- & ---- & ---- \\
\hline \multirow{2}{*}{$\begin{array}{c}\text { Positive } \\
\text { Control } \\
\text { (G II) }\end{array}$} & Mean \pm SE & $24.61 \pm 0.28^{c}$ & $3.86 \pm 0.1^{b}$ & $16.07 \pm 0.17^{\mathrm{a}}$ \\
\hline & \% Change & 102.47 & 217.84 & 290.38 \\
\hline \multirow{2}{*}{$\begin{array}{l}\text { Se (C) } \\
\text { (G III) }\end{array}$} & Mean \pm SE & $1.44 \pm 0.07^{\mathrm{g}}$ & $1.11 \pm 0.03^{\mathrm{d}}$ & $3.08 \pm 0.26^{\mathrm{f}}$ \\
\hline & \% Change & -88.12 & -9.09 & -25.20 \\
\hline \multirow{2}{*}{$\begin{array}{l}\text { Se (W) } \\
(\mathbf{G ~ I V})\end{array}$} & Mean \pm SE & $1.20 \pm 0.17^{\mathrm{g}}$ & $0.62 \pm 0.07^{\mathrm{e}}$ & $8.85 \pm 0.46^{\mathrm{d}}$ \\
\hline & \% Change & -95.14 & -84.06 & -44.91 \\
\hline
\end{tabular}

The means \pm SE followed by the different letter in groups are significantly from each other with probability level $(\mathrm{P} \leq 0.05)$. 


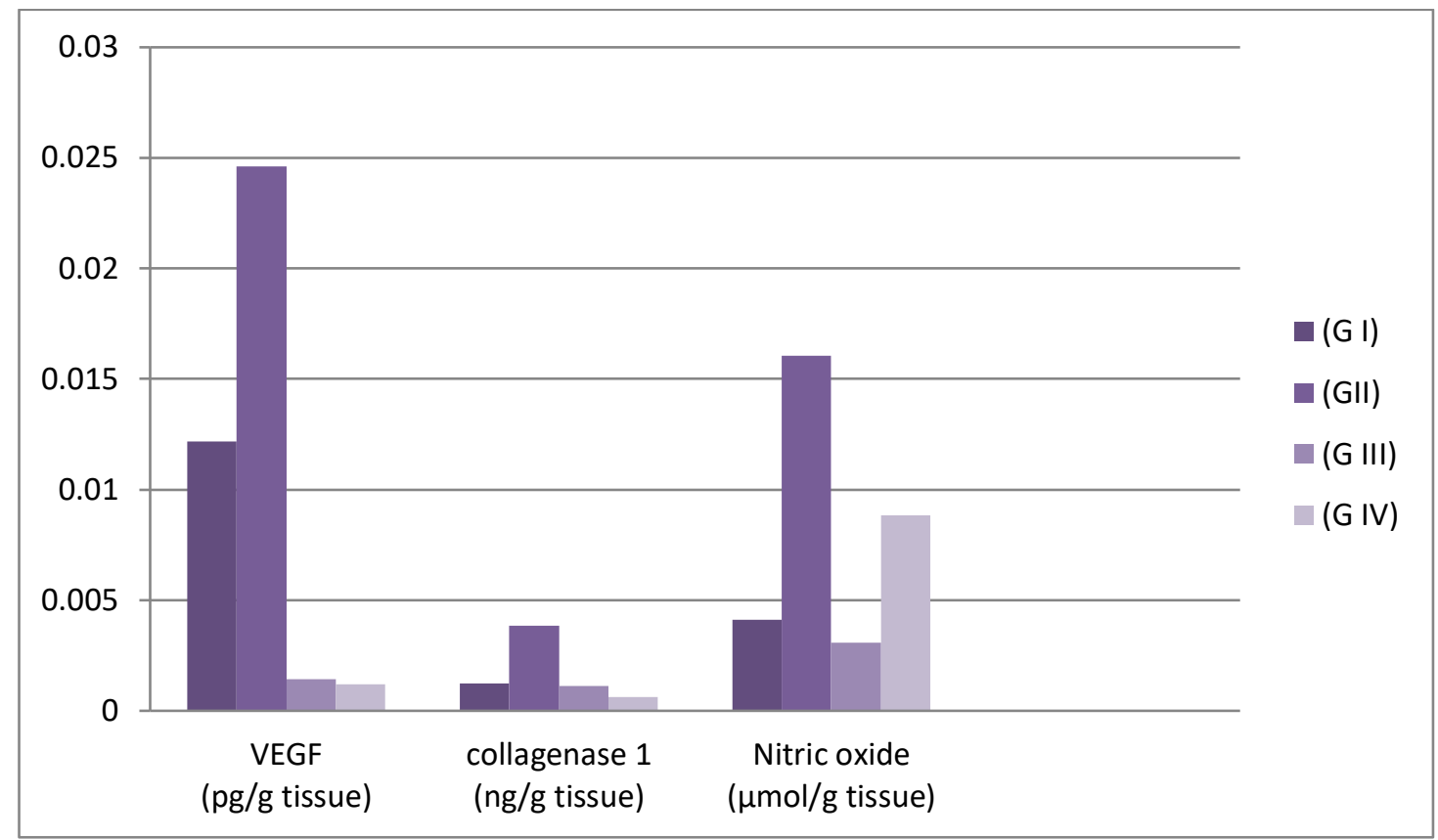

Figure (4): Vascular endothelial growth factor levels, Collagenase 1 activity and

Nitric oxide (NO) concentrations in skin tissues of all studied groups.

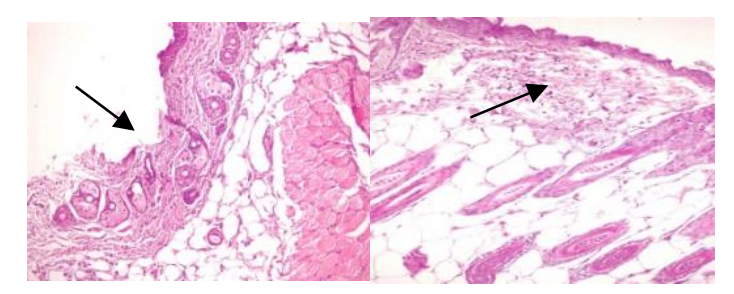

A

B

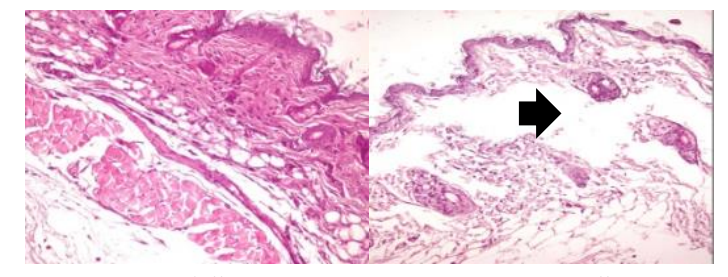

A"

B"

Figure (5): (H \& $\mathrm{E} \times 400)$ staining of sections from skin tissue of female albino mice. Where (A): (G II), showing epidermal ulceration; note the detached epidermal layer with brocken basement membrane (arrow). (B):(G IV), showing focal leucocytes cells infiltrations in the dermal layer (arrow). (But (A"): (G I) showing normal skin layers; note the normal epidermis and dermis. (B"): (G III), showing intact epidermis with edema in the dermis layer; note the increased thickness in the dermis together with dispersed tissue elements (arrow head). 